\title{
STABILITY AND SURFACE FREE ENERGY ANALYSIS OF A LIQUID DROP ON A HORIZONTAL CYLINDRICAL WIRE USING FEM SIMULATION
}

\author{
Prabin Haloi $^{1}$, Rupam Baruah ${ }^{2}$, Numan Siddique Mazumder ${ }^{3}$, Dhrubajyoti Sarma ${ }^{4}$, Amrendra Bharti ${ }^{5}$ \\ ${ }^{1}$ Assistant Professor, Department of Mechanical Engineering, Tezpur University, Assam, India \\ ${ }^{2}$ Graduated Student, Department of Mechanical Engineering, Tezpur University, Assam, India \\ ${ }^{3}$ Graduated Student, Department of Mechanical Engineering, Tezpur University, Assam, India \\ ${ }^{4}$ Student, Department of Mechanical Engineering, Tezpur University, Assam, India \\ ${ }^{5}$ Student, Department of Mechanical Engineering, Tezpur University, Assam, India
}

\begin{abstract}
The stable conformation of a liquid-water drop on a horizontal cylindrical wire is studied. The stable conformation is established with various wire diameters. For each conformation of the drop the surface free energy is calculated using FEM simulation. The free energy for different drop shapes are compared with consideration to different volumes and contact angles. The effect of gravity on droplet shape with respect to wire diameter and droplet volume is observed. The droplet configurations under the influence of gravity and in the absence of gravity are observed and the shapes are described through coordinates of liquid-air and liquid-solid interfaces. The compensation of one interfacial energy with that of another is found. The wetting behaviour of liquid-drop on a wire is found to be significantly different from that on a plane surface under the influence of wire geometry. The numerical model was established taking liquid density at 1000 kg/m3 and gravitational acceleration at $9.8 \mathrm{~m} / \mathrm{s}^{2}$. The numerical study is important in understanding the spreading of liquid droplets over cylindrical surfaces .The spreading of liquid over surfaces has got wide application in food industry, micro fluidics and more and in understanding the coating behaviour, liquid-solid and liquid-vapour interactions, material properties, etc.
\end{abstract}

Keywords: Surface Free Energy, FEM Simulation, Contact angle, Gravity ***

\section{INTRODUCTION}

The motion of a liquid drop under the combined influence of surface tension, gravitational, and viscous forces has received many attention in the fluid mechanics problems. Fluid flow over surfaces has many industrial applications, such as coating of objects to form films and also heat transfer related problems, in human lungs to form a thin mucous film. It is also a topic of fundamental interest as it provides information on the interaction between the solid-liquid, solid vapor and liquid vapor interfaces. Solid cylindrical surfaces have got importance related to composite materials and helps to evaluate interfacial energy and in understanding the material as well as the wire interface properties. Droplets sliding down a vertical wire and encountering an intersection have been employed to perform a blocking/crossing sharp transition, which is the basic component of wire-based microfluidic devices. It is seen that the behavior of a droplet on a wire plays a key role in digital micro fluidics on a wire. The wetting phenomena of liquid drops on wire have been reported in determining the drop's shape and on extracting the contact angle accurately.
In our present work, we consider the motion of a liquid drop on a cylindrical wire oriented with the axial direction perpendicular to gravity. The aim of this work is to analyze the behavior of a liquid droplet on a horizontal cylindrical wire for different volume and contact angles. The shape and stability of a drop of water on the wire for different volume and contact angles are determined. The calculations are done taking different wire radius and in the presence of gravity and finally numerical simulation of the problem is performed using Finite element simulation technique.

Laplace's law is seen to be used to get an idea on excess pressure across curved surfaces that help to know about capillarity effects, and the idea of interfacial tension gives the free energy required to increase area of contact between two phases. The excess pressure allows calculating the mechanical equilibrium for the droplet shape, whereas the free energy determines the contact area between two phases.

The wetting behavior of a droplet-on-wire is seen to be quite different from that of a droplet on a plane surface. This has been mainly attributed to the wire geometry. 


\section{METHODOLOGY}

\subsection{Mechanism of Flow}

Spreading of a liquid droplet on a wire follows a different mechanism compared to surfaces that are not curved depending on geometry. De Gennes [1] examined the formation of film and macroscopic droplet on a wire and flat surfaces taking the sign of spreading power. De Ruijter et al [2] studied the reasons of wetting of wires and the important of carbon nanotubes. Carroll et al [3] used elliptic integrals to determine the metastabillity conditions of barrel shaped droplets with variation in the volume and contact angle. The transition between clam shell and barrel-shape was studied by Adam et al [4] to find a relation between detergent action and emulsification. McHale and Newton [5] compared the surface free energy for to conformations with same drop volume or contact angle. The control of the equilibrium waiting by modifying local and surface geometry was experimentally demonstrated by Richard and Quere [6]. Carroll [7] studied the change of Laplace excess pressure at a constant drop volume when a barrel-shape droplet is subjected to a small perturbation. The existence of an inflection point in the profile of a barrel-shape droplet was also examined by McHale [8].

In contrast to the barrel shape droplet problem, no solution to Laplace's equation for the asymmetric clam-shell shape is reported except for such numerical approaches as finite element methods [5,8]. Gilet et al [9] discussed the roll-up transition (barrel to clam-shell) in the wetting process on a wire. Work with respect to gravitational distortion of Barrelshape droplets on vertical wires was studied by Gilet et al [10]., A microscopic study of the effect of wire orientation on the wire wetting process subjected to gravity was studied by Mullins et. al. [11] and tried to represent the heterogeneous nature of fibrous materials in the wetting process.

The hydrophilic and hydrophobic of surfaces have been studied to understand the slip behavior of water. The contact angle for a hydrophobic surface causes water to bead up while a hydrophilic surface allows a drop of liquid to spread out. The merging and splitting of liquid drops due to affinity is affected by the fluid-solid interfaces. A curved surface helps the force due to surface tension eliminate the pressure force.

The barrel-shape (low contact angle) is axisymmetric while the clam-shell shape (high contact angle) is asymmetric. Deformation of droplet shape with respect to droplet volume and gravity is analyzed.

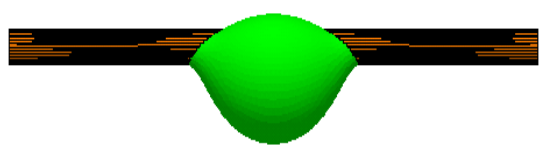

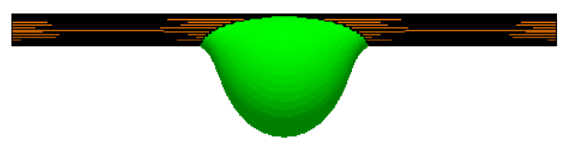

Fig 1: Gravity effect on a water drop
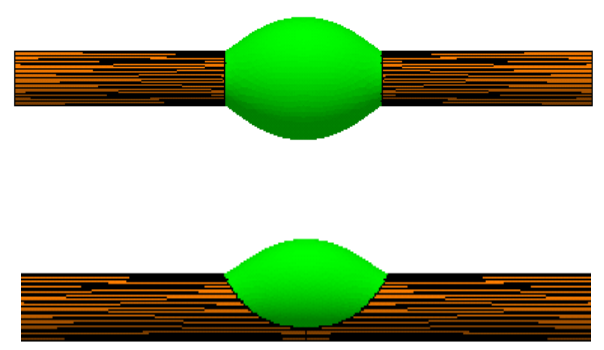

Fig 2: Barrel and Clam-shell shape

\subsection{Equilibrium Condition of Water Droplet}

The energetically preferred shape or conformation is necessary to determine the equilibrium shape of each of the barrel and clam-shell shape. The absolute stability condition can then be determined (Carroll). The surface free energy of each of the two conformations is needed to determine the drop stability on a wire. The effect of gravity has to be considered along with different volume and contact angles as cited by researchers in their works.

In the present work, two initial shapes were developed for each droplet volume and contact angles. One drop sits on the wire placed horizontally while the other droplet is wrapping around the wire. The former developed a clam-shell shape whereas the later tends towards a barrel-shape. The stable configuration that can sustain perturbation may be from either of one of the two initial conditions or it may be both. The basic geometries for the wire surface during simulation were the edges, vertices or facets were used.

\subsection{Numerical Simulation}

Finite element Simulation has been used to obtain different parameters such as surface free energy, liquid-solid and liquidvapor interfacial areas, capillary length and the Laplace pressure outside the droplets. The initial setup is made for the two conformation using FEM simulation. The surface energies of different conformations are determined for different volume and contact angles. A comparative analysis is made for the reduced volume droplets at a constant wire radius and varying the contact angle. 


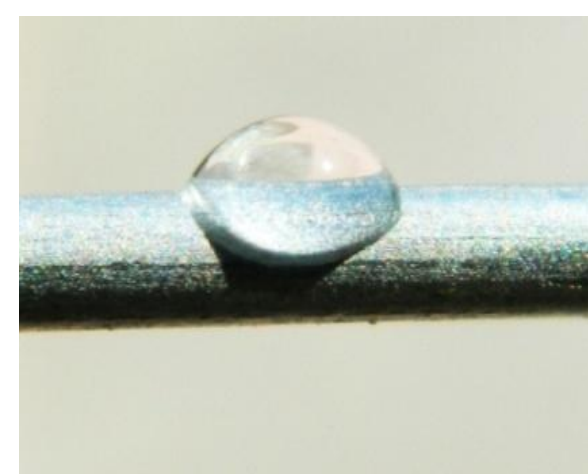

Fig 3: Stable clam shell shape

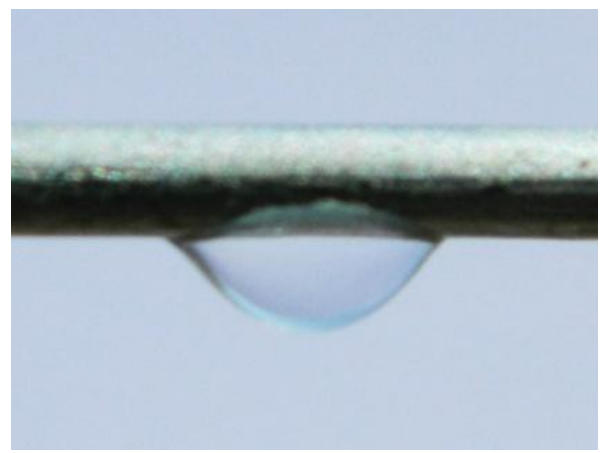

Fig 4: Downward clam shell shape

\section{RESULTS AND DISCUSSION}

In the present work, theoretical model of equilibrium shape of a water droplet on cylindrical solid wire surfaces were established and the equilibrium shape of droplet was simulated by FEM simulation. The surface energies for droplet conformation are calculated through the simulation. The shape of the droplet is described through the coordinate of nodes on the liquid-air interface. The height and diameter of the contact area of equilibrium droplet is obtained from the data. For calculation purpose liquid density, gravitational acceleration and the liquid-air interfacial energy are assumed to be 1000 $\mathrm{kg} / \mathrm{m} 3,9.8 \mathrm{~m} / \mathrm{s} 2,0.07275 \mathrm{~J} / \mathrm{m} 2$. The simulations were performed by Surface Evolver to evolve the drop configuration from the initial model to the one with minimum system energy. Calculations were done to arrive at minimum system energy and are assumed within the limit of 104 . Our calculation was performed until the total free energy difference converges to within the acceptable tolerance of 104 when we considered it is at minimum system energy. For drop volume $\mathrm{V}=4{ }^{\mu} \mathrm{L}$ and Contact angle $\theta=250$ for a wire of radius $\mathrm{a}=100 \mu \mathrm{m}$. We simulate the shapes of the droplet on the wire using surface evolver FEM simulation.

By using FEM simulation we determine the values of surface energy for different volume with wire radius $100 \mu \mathrm{m}, 125^{\mu}$ $\mathrm{m}$ and $150 \mu \mathrm{m}$.
For $\mathbf{r}=100 \mu m$

Table1: Volume and free energy for $\mathrm{r}=100 \mu \mathrm{m}$

\begin{tabular}{|ll|}
\hline Volume $(\mathrm{L})$ & Energyx $10-8(\mathrm{~J})$ \\
\hline 0.05 & 3.34 \\
\hline 0.10 & 5.63 \\
\hline 0.15 & 7.62 \\
\hline 0.20 & 9.44 \\
\hline
\end{tabular}

For $\mathbf{r}=125 \mu$

Table 2: Volume and free energy for $\mathrm{r}=125^{\mu} \mathrm{m}$

\begin{tabular}{|ll|}
\hline Volume (L) & Energyx10-8(J) \\
\hline 0.05 & 3.14 \\
\hline 0.10 & 5.33 \\
\hline 0.15 & 7.23 \\
\hline 0.20 & 8.98 \\
\hline
\end{tabular}

For $\mathbf{r}=150 \mu$

Table 3: Volume and free energy for $\mathrm{r}=150 \mu \mathrm{m}$

\begin{tabular}{|cc|}
\hline Volume (L) & Energyx10 $^{-8}(\mathbf{J})$ \\
\hline $\mathbf{0 . 0 5}$ & 2.90 \\
\hline $\mathbf{0 . 1 0}$ & 5.06 \\
\hline $\mathbf{0 . 1 5}$ & 6.90 \\
\hline $\mathbf{0 . 2 0}$ & 8.58 \\
\hline
\end{tabular}

Plotting these surface energy data against volume we get

\section{For $\mathbf{r}=100{ }^{\mu} \mathbf{m}$}

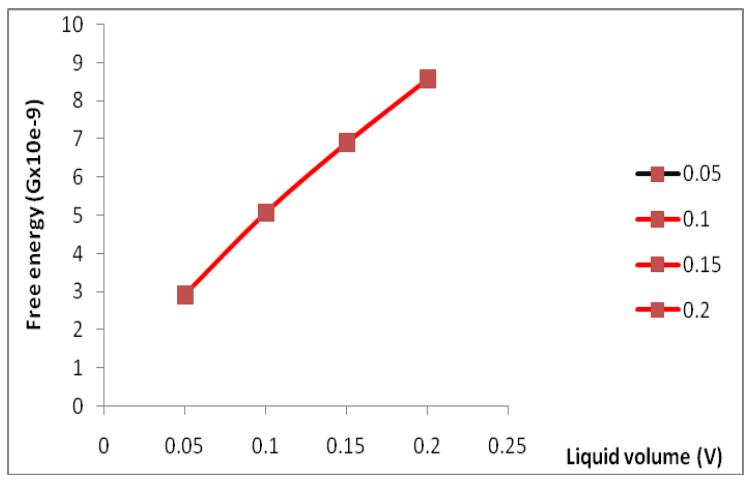

Graph 1: Free energy v/s liquid volume for $\mathrm{r}=100^{\mu} \mathrm{m}$ 


\section{For $\mathbf{r}=125 \mu \mathrm{m}$}

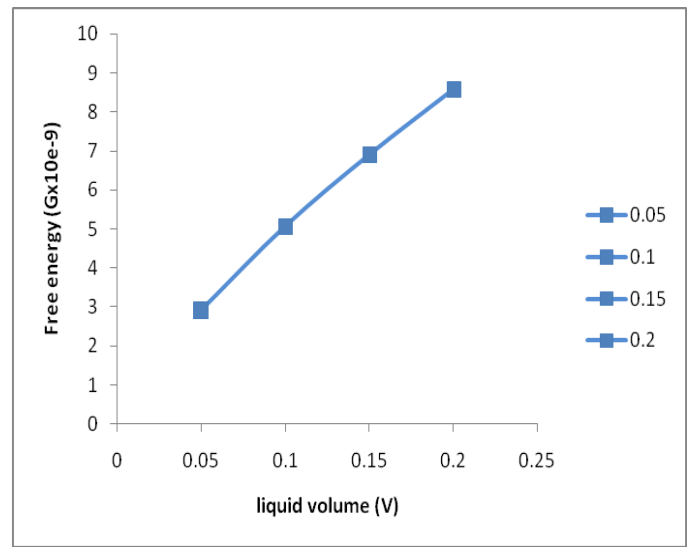

Graph 2: Free energy v/s liquid volume for $\mathrm{r}=125 \mu \mathrm{m}$

\section{For $\mathbf{r}=150{ }^{\mu} \mathbf{m}$}

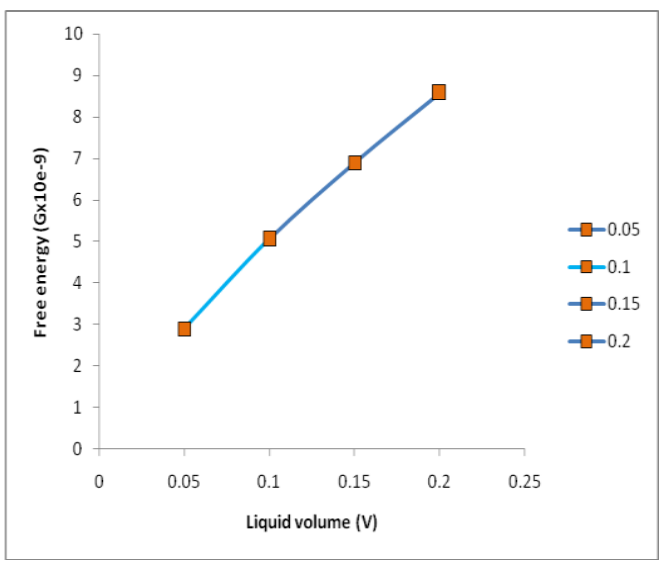

Graph 3: Free energy v/s liquid volume for $\mathrm{r}=150^{\mu} \mathrm{m}$

From the above plots, it has been proved that when the liquid volume of droplet increases, the free surface energy also found to be increased. So, for higher volume of liquid drops the barrel configuration does not appear to exist. The reason for this can again be attributed to the fact that increase in the interfacial energy for a solid-liquid interface is not equivalent to the loss in gas-liquid interface and the energy loss due to gravitational effects.

Simulation for a constant liquid droplet volume is carried out with constant wire radius $(\mathrm{r}=100 \mu \mathrm{m})$. the possibilities of formation of a barrel shaped droplet with variation in contact angle has been observed. It has been found that an increase in contact angle between solid-liquid results in a decrease in barrel shape formation but the tendency of formation of downward clam- shell shaped droplet is increased.
At a constant value of wire radius, $\mathrm{r}=100 \mu \mathrm{m}$, different values of contact angle $\theta=200,250,300,350,400,500$ and 600 are considered to obtain droplet shaped and the free energy.

Table 4: Shapes and free energy for $\mathrm{r}=100 \mu \mathrm{m}$ with varying contact angle

\begin{tabular}{|l|l|l|}
\hline $\begin{array}{l}\text { CONTACT } \\
\text { ANGLE }\end{array}$ & SHAPE & $\begin{array}{l}\text { FREE } \\
\text { ENERGY } \quad \times \\
10-7\end{array}$ \\
\hline 200 & 2.63 \\
\hline 250 & & 2.67 \\
\hline 300 & & 2.70 \\
\hline 350 & & 2.73 \\
\hline 400 & & 2.76 \\
\hline 500 & & 2.88 \\
\hline 600 & & \\
\hline
\end{tabular}

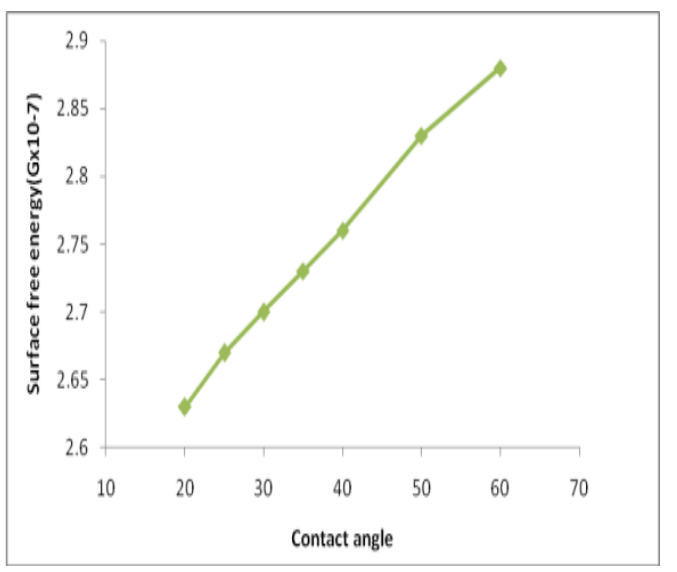

Graph 4: Contact Angle V/S surface energy

A phase diagram is developed for the droplet shape with respect to contact angle and reduced volume of the liquid using FEM simulation. For a constant wire radius the stability for different conformation is determined 


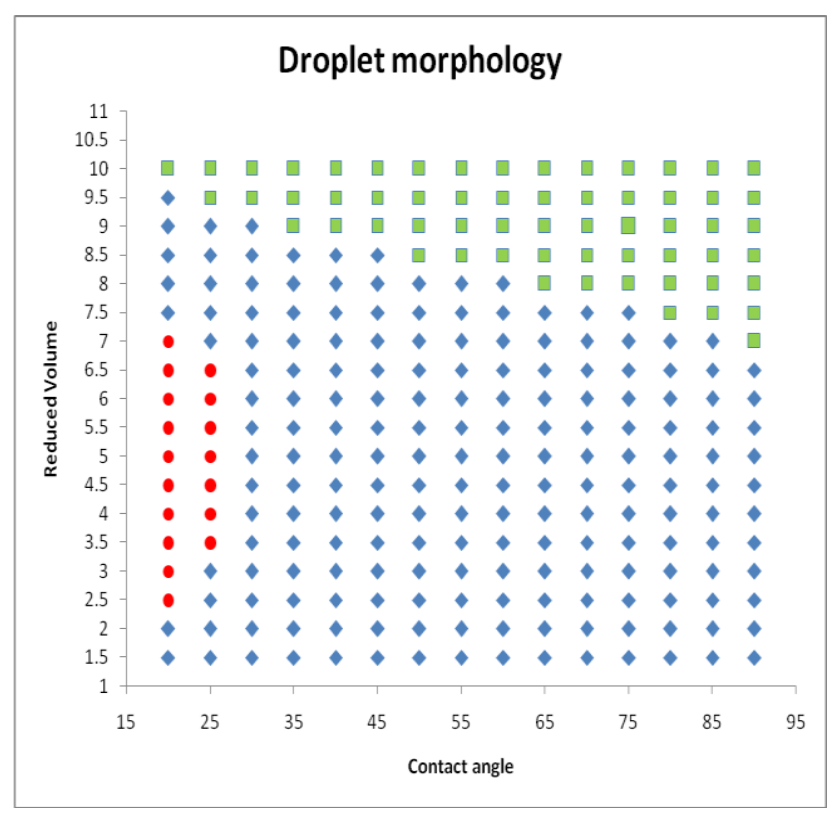

Graph 5: reduced volume v/s contact angle

In the above graph color coding is used to distinguish the following

For falling off (green)

For coexistence of both conformation (red)

For downward clam shell conformation (blue)

The region of coexistence is seen to be very small. For higher liquid volume or high contact angle the barrel conformation is not stable. The number of downward clam-shell shaped conformation is relatively high. The droplet seems to detach from the wire when the gravitational force break the droplet pinning along the contact line. Increase in gravity force helps to expand the space for clam-shell, thereby reducing the region of barrel stability.

\section{CONCLUSIONS}

Numerical models are used to establish equilibrium shapes on solid cylindrical surfaces followed by computerized simulation. Free surface energy calculations so determined helps in obtaining the droplet morphology. Thus an initial investigation as the present analysis will be useful to understand the dynamic flow behavior of liquid drops on solid cylindrical surfaces. The various estimation found can be useful to materialists and surface designers to find better flow surface materials and types of film formation. Further research will be required for proper estimation of microscopic droplet flow over different surface geometries.

\section{REFERENCES:}

[1]. De Gennes, P.G. (1985) Wetting: Statics and Dynamics. Rev. Mod. Phys., 57, 827-863.

[2]. De Ruijter, M.J., Blake, T.D. and De Coninck, J. (1999) Dynamic Wetting Studied by Molecular Modeling Simulations of Droplet Spreading. Langmuir, 15, 7836-7847

[3]. Carroll, B.J. The Accurate Measurement of Contact Angle, Phase Contact Areas, Drop Volume, and Laplace Excess Pressure in Drop-on-Fiber Systems Langmuir 1986, 2, 248

[4]. Adam, N. J. Soc. Detergent Action and its relation to wetting and emulsification Dyers Color. 1937, 53, 121

[5]. McHale, G.; Newton, M. I.; Carroll, B. J.The Shape and Stability of Small Liquid Drops on Fibers Oil Gas Sci. Technol. 2001, 56, 47

[6]. Richard, D. and Quéré, D. (1999) Viscous Drops Rolling on a Tilted Non-Wettable Solid. Europhys.Lett.,48, 286-291. [7]. Carroll, B. J.Equilibrium conformation of liquid drops on thin cylinders under forces of capillarity .Colloid Interface Sci. 1976, 57, 488

[8]. McHale, G.; Newton.Global geometry and the equilibrium shapes of liquid drops on fibers M. I. Colloids Surf. A 2002, 206, 79

[9]. Gilet, T.; Terwagne, D.; Vandewalle, N. Digital microfluidics on a wire. Appl. Phys. Lett. 2009, 95, 014106

[10]. Gilet, T.; Terwagne, D.; Vandewalle, N. Droplets sliding on fibres. Eur. Phys. J. E 2010, 31, 253-262

[11]. Mullins, B. J.; Agranovski, I. E.; Braddock, R. D.; Ho, C. M. Effect of wire orientation on wire wetting processes. J. Colloid Interface Sci. 2004, 269, 449-458

[12]. Eral, H. B.; de Ruiter, J.; de Ruiter, R.; Oh, J. M.; Semprebon, C.; Brinkmann, M.; Mugele, F. Drops on functional wires: From barrels to clamshells and back. Soft Matter 2011, 7, 5138-5143

[13]. Carroll, B. J. Equilibrium conformations of liquid-drops on thin cylinders under forces of capillarity - a theory for the roll-up process. Langmuir 1986, 2, 248-250

[14]. Brakke, K. Surface evolver .Exp. Math. 1992, 1, 141

[15]. P. Contal, J. Simao, D. Thomas, T. Frising, S. Calle, J.C. Appert-Collin, D Bemer, Clogging of fiber filters by submicron dropletJ Aerosol Sci. 35 (2004) 263

[16]. Léger, L. and Joanny, J.F. (1992) Liquid Spreading. Rep. Prog. Phys., 55, 431-486.

[17]. Neinhuis, C.; Barthlott, W., Characterization and distribution of water-repellent, self-cleaning plant surfaces. Annals Botany 1997, 79, (6), 667-677.

[18]. Hoefnagels, H. F.; Wu, D.; de With, G.; Ming, W., Biomimetic superhydrophobic and highly oleophobic cotton textiles. Langmuir 2007, 23, (26), 13158-13163

[19]. Quéré, D. (1999) Fluid Coating on a Fiber. Ann. Rev. Fluid Mech., 31, 347-384

[20]. Ren H W, Xu S, Wu S T. Effects of gravity on the shape of liquiddroplets. Opt Commun, 2010, 283(17): 3255-3258. 


\section{BIOGRAPHIES:}

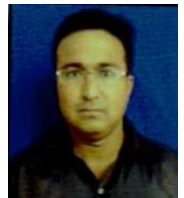

P. Haloi currently works as Assistant Professor of Mechanical Engineering at Tezpur University, Assam. He also served as a Senior Engineer in Indian Railways. His research interests are in Wind Engineering and Fluid

Flow.

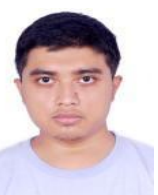

Rupam Barua is a graduate student in Mechanical Engineering. He graduated in Bachelor of Technology in Mechanical Engineering, Tezpur University .

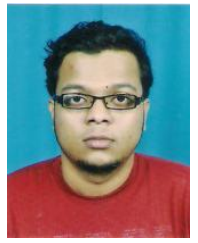

Numan Siddique Mazumder is a graduate student in Mechanical Engineering. $\mathrm{He}$ graduated in Bachelor of Technology in Mechanical Engineering, Tezpur University .

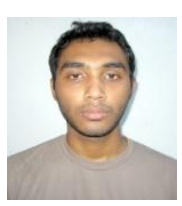

Dhruba Jyoti Sarma is a final year student in Bachelor of Technology in Mechanical Engineering, Tezpur University.

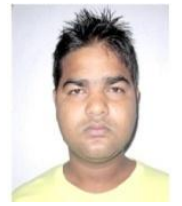

A. Bharati is a final year student in Bachelor of Technology in Mechanical Engineering, Tezpur University. 